\title{
4-D PET joint image reconstruction/non-rigid motion estimation with limited MRI prior information
}

\author{
Alexandre Bousse ${ }^{1 *}$, Jieqing Jiao ${ }^{2}$, Kjell Erlandsson ${ }^{1}$, Luis Pizarro², Kris Thielemans ${ }^{1}$, Dave Atkinson², \\ Sébastien Ourselin², Simon Arridge ${ }^{2}$, Brian Hutton ${ }^{1,3}$ \\ From PSMR14: 3rd Conference in PET/MR and SPECT/MR \\ Kos Island, Greece. 19-21 May 2014
}

${ }^{1}$ Institute of Nuclear Medicine-UCL, University College London, London NW1 2BU, UK

Motion compensated gated PET image reconstruction methods include jointreconstruction (JR) and indirect reconstruction (IR) with pre-estimated motion from MRI (MRI-IR). JR suffers from poor PET data quality whereas MRI-IR requires highquality MRI volumes at each gate. We propose a penalised maximum-likelihood approach combining JR and MRI-IR. Our method is referred to as minimal MRI prior JR (MP-JR).

The $M$ gates data are stored in $\boldsymbol{g}=\left[\boldsymbol{g}_{1} ; \ldots ; \boldsymbol{g}_{M}\right]$ where $\boldsymbol{g}_{m}$ is the measurement vector at gate $m$. Each $\boldsymbol{g}_{m}$ is a Poisson distributed vector of parameter $\overline{\mathrm{g}}\left(\mathrm{f}, \alpha_{m}\right)=\mathrm{P} W\left(\alpha_{m}\right) \mathrm{f}+\mathrm{r}_{m}$ where $\boldsymbol{P}$ is the projector, $W\left(\boldsymbol{\alpha}_{m}\right)$ is the $m$-th motion of parameter $\boldsymbol{\alpha}_{m}, \boldsymbol{r}_{m}$ is the $m$-th average random/scatter vector and $\boldsymbol{f}$ is the activity at $m=1$. JR is achieved with (1).

$$
\text { minimise } L(\mathrm{f}, \alpha)=-\sum_{m=1}^{M} \sum_{i=1}^{I} g_{i, m} \log \bar{g}_{i}\left(\mathrm{f}, \alpha_{m}\right)+\bar{g}_{i}\left(\mathrm{f}, \alpha_{m}\right) \text { w.r.t. f, } \alpha
$$

MRI-IR is achieved by solving (2)

$$
\text { minimise } L\left(\mathrm{f}, \alpha^{m r i}\right) \text { w.r.t. } \mathrm{f}
$$

MP-JR is achieved with (3).

$$
\text { minimise } L(\mathrm{f}, \alpha)+\beta \sum_{\ell \in \mathcal{S}}\left\|\alpha_{\ell}-\alpha_{\ell}^{m r i}\right\|_{2}^{2}+\gamma \sum_{m=1}^{M-1}\left\|\alpha_{m+1}-\alpha_{m}\right\|_{2}^{2}
$$

The first term accounts for PET data, whereas the second term accounts for MRI motion information from subset $S$. The last term controls temporal smoothness.

We tested each method on 9 PET FDG volumes generated from a real dynamic MRI sequence. Tumours were added to the activity distribution (invisible in the MRI). The gates subset $S$ for MP-JR contains the reference gate, end-inspiration and end-expiration. Reconstruction profiles 1 show that MRI-IR improves edges visible in the MRI but degrades the tumours. On the contrary, JR performs well on tumours, but the

\section{SpringerOpen ${ }^{\circ}$}

(C) 2014 Bousse et al; licensee Springer This is an Open Access article distributed under the terms of the Creative Commons Attribution License (http://creativecommons.org/licenses/by/4.0), which permits unrestricted use, distribution, and reproduction in any medium, provided the original work is properly cited. 


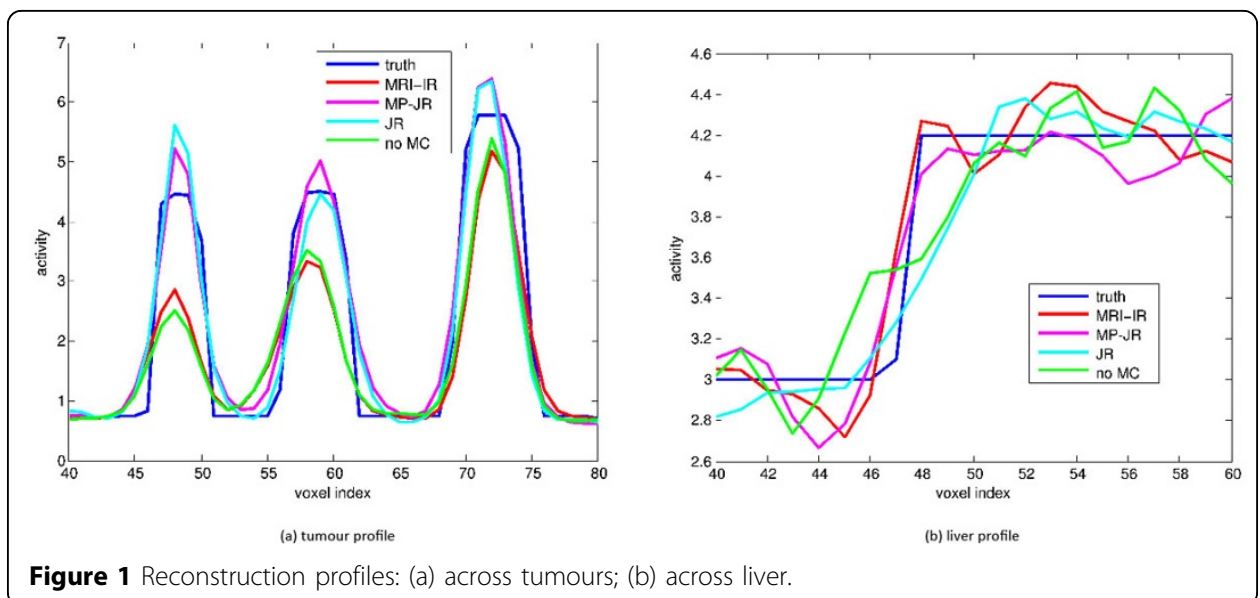

edges are poorly reconstructed. MP-JR appears to perform well on both organ edges and tumours.

MP-JR seems to perform well where both JR and MRI-IR under-perform. This is due to the fact that MP-JR relies on both MRI and PET data. In addition, results tend to show that with temporal smoothing on B-spline parameters, a subset of MRI volumes provides sufficient information.

\section{Acknowledgements}

This work was supported by UK EPSRC (EP/K005278/1). UCL/UCLH research is supported by the NIHR BRCs funding scheme.

\section{Authors' details}

IInstitute of Nuclear Medicine-UCL, University College London, London NW1 2BU, UK. ${ }^{2}$ Centre for Medical Image Computing, University College London, London WC1E 6BT, UK. ${ }^{3}$ Centre for Medical Radiation Physics at the University of Wollongong, NSW, Australia.

Published: 29 July 2014

\section{Submit your manuscript to a SpringerOpen ${ }^{\odot}$ journal and benefit from:}

- Convenient online submission

- Rigorous peer review

- Immediate publication on acceptance

- Open access: articles freely available online

- High visibility within the field

- Retaining the copyright to your article 